CARDIOVASCULAR MEDICINE

\title{
Survival differences between heart failure in general practices and in hospitals
}

\author{
O Wendelboe Nielsen, J Hilden, T McDonagh, J Fischer Hansen
}

Heart 2003;89:1298-1302

See end of article for authors' affiliations

....................

Correspondence to: Dr O W Nielsen,

Cardiology Department B,

Rigshospitalet, 2200

Copenhagen, Denmark;

own@dadlnet.dk

Accepted

27 February 2003
Objectives: To compare the survival of patients thought to have heart failure in general practice (GP-HF) with that of patients with heart failure in hospital (hospital-HF), patients with heart disease but without heart failure (non-HF), and a control group without heart disease.

Design and setting: Cross sectional study from general practice with a prospective follow up from 5.3 to 7.4 years.

Participants: 2157 community participants, 1999 of whom lived outside nursing homes, were selected because they were registered with four general practitioners at entry. Study participants were divided into the four groups after a review of general practice case notes, questionnaires, and interviews.

Main outcome measures: Five year survival and multivariate predictors of all cause mortality.

Results: Five year survival was $76 \%$ in the control group ( $n=571$, mean age at entry 74.1 years), $71 \%$ in non-HF patients ( $\mathrm{n}=218,74.4$ years), $61 \%$ in GP-HF patients ( $\mathrm{n}=67,75.8$ years), and $39 \%$ in hospital-HF patients ( $n=33,76.7$ years). The median survival times were 6.8 years for GP-HF patients and 3.9 years for hospital-HF patients. Significant predictors of mortality in the multivariate Cox model of 1979 patients living outside nursing homes were hospital-HF (hazard ratio (HR) 2.1, p = 0.002), GP-HF (HR 1.7, $p=0.004)$, non-HF heart disease (HR 1.4, $p=0.03$ ), previous myocardial infarction (HR 1.6, $p=0.04)$, no response to questionnaire (HR 2.0, $p<0.0001$ ), higher age (for every 10 years, HR 2.4, $p<0.0001$ ), and male sex (HR 2.1, $p<0.0001)$. Other factors such as atrial fibrillation, hypertension, and diabetes were not significant.

Conclusion: Heart failure in general practice is associated with a worse survival than that seen in the control group but is better than for patients who have been treated at least once for heart failure in a hospital.
$\mathrm{T}$ he survival of patients discharged from hospital with a diagnosis of heart failure is well described, but much less is known of unselected patients thought to have heart failure in general practice (GP-HF). Population studies of volunteer participants have described a five year survival of $59 \%$ in Rotterdam ${ }^{1}$ and $74 \%$ in 60 year old men from Gothenburg. ${ }^{2}$ The 10 year survival was $64 \%$ in the NHANES (national health and nutrition examination survey) study of patients in the USA aged 25-74 years. ${ }^{3}$ Other studies used a more definite diagnosis of heart failure and found a much poorer survival with only $70-75 \%$ alive after one year in Framingham, Rochester, and London. ${ }^{4-6}$ As heart failure is often diagnosed on loose criteria in general practice ${ }^{7-10}$ the patients may be different from those seen in hospital and clinical trial settings. It is not known whether unselected GPHF patients have a different survival from patients with heart failure who are managed in hospital (hospital-HF), patients with heart disease but without heart failure (non-HF), or a matched control group. Since $6.4 \%$ of the population $>50$ years of age have received treatment for possible or definite heart failure ${ }^{9}$ it would be helpful to point out those with the highest risk.

The main aim of this study was to compare survival among GP-HF patients with that of patients treated for heart failure in hospital at least once and with the survival among patients with non-heart failure heart disease and among control participants. Secondly, we aimed at identifying clinical mortality predictors among patients thought to have heart failure in the community.

The target of our project is patients living outside nursing homes, which in Denmark means patients who are severely mentally and physically disabled. Nursing home residents were therefore excluded from the survival analyses but readily accessible baseline data are reported as a byproduct.

\section{METHODS \\ Population}

The survey methods have been presented in earlier publications. ${ }^{911}$ The cross sectional study was carried out in 2158 community patients who were registered with three general practices at three different dates in 1993 to 1996. They were selected independently of previous health contacts and as all participants $\geqslant 50$ years of age from three of the practices $(\mathrm{n}=1754)$ and all participants aged $40-49$ years from one of the practices $(n=403)$. The total was reduced to 2157 after deletion of a duplicate record. The age and sex composition in the study population was not different from that of the entire Copenhagen Municipality, and there was no difference in relevant socioeconomic parameters between the studied districts and the entire Copenhagen Municipality. The study was approved by the local ethics committee (appraisal No 01-086/95).

\section{Cross sectional screening procedure}

A research registrar in cardiology (OWN) screened all general practice case notes for information about cardiac disease. Further information was obtained from hospital discharge

Abbreviations: $\mathrm{Cl}$, confidence interval; GP-HF, heart failure in general practice; hospital-HF, heart failure in hospital; HR, hazard ratio; NHANES, national health and nutrition examination survey; non-HF, heart disease without heart failure 
letters, telephone interviews, and in some cases from hospital records.

\section{Questionnaire}

A questionnaire was sent to all participants outside nursing homes $(n=1999)$. It asked for details about breathlessness, chest pain, and past heart disease. Breathlessness required confirmative answers to at least one of the Medical Research Council breathlessness questions. Questions about breathlessness when hurrying or breathlessness at night were discarded..$^{12}$ Participants in the second and third practice also gave details of physician diagnosed angina, myocardial infarction, fluid in the lungs, treatment for peripheral fluid retention, chronic obstructive airways disease (chronic bronchitis or asthma), diabetes, stroke, and treatment with cardiac drugs. Non-responders received a single reminder. The questionnaire response rate was $86 \%$ (1504 of 1756) for participants $<80$ years of age, and $48 \%$ (191 of 401 ) of participants $>80$ years were either non-responders or residing in nursing homes.

\section{Definition of study groups}

On the basis of case note reviews, discharge letters, and interviews, 357 patients had some indication of heart disease: 115 with heart failure and 242 with non-HF heart disease. Matched control participants were randomly selected from the remaining 1800 participants. Definitions were as follows.

Heart failure in hospital (hospital-HF group) was identified in 38 patients with at least one recorded episode with signs and symptoms of heart failure that were managed in a hospital.

Heart failure in general practice (GP-HF group) was identified in 77 patients with a recorded episode of treatment for signs and symptoms suggestive of heart failure. The signs and symptoms were comparable with "possible or definite heart failure" in the Boston index. ${ }^{913}$

Heart disease without heart failure (Non-HF group) applied to patients $(\mathrm{n}=242)$ with a myocardial infarction during hospitalisation; angina pectoris based on typical chest pain and antianginal treatment; atrial fibrillation when documented by an ECG; suspected heart disease (unexplained cardiac symptoms, treatment, admissions, and abnormal electrocardiographic or radiographic changes); or hypertensive and diabetic heart disease (noted if the patient had suspected heart disease as well as diabetes or hypertension).

Control participants (control group) were randomly selected from among those without heart disease or heart failure. One or two control participants were picked for each of the 357 patients. They were matched according to sex, registration with the same general practitioner, age \pm 2 years, and preferentially whether they had responded to the questionnaire. According to these rules, two matched control participants were identified for 316 patients, one control for 40 patients, and none for one 80 year old woman with heart disease without heart failure. The group thus comprised 672 participants, leaving 1138 (2157 - $357-672)$ not selected.

\section{Follow up}

Flagging each patient's record with the Central Personnel Register for Denmark on 8 March 2001 identified the date of death. Information about end points was missing for 20 participants without heart disease or heart failure at baseline.

\section{Statistical analysis}

Differences in clinical features between patients with heart failure, patients with heart disease, and the control group were evaluated by the $\chi^{2}$ test using the Yates continuity correction or Student's $t$ test. Survival was estimated with the method of Kaplan and Meier. Differences in survival between groups were evaluated by the log rank test. Two tailed tests of significance are reported. The associations between clinical features and observed mortality were examined by Cox proportional hazards modelling. All of the covariates with $\mathrm{p}<0.15$ in the univariate regression model were initially entered into a multivariate model and discarded one by one if they did not add any significance $(p<0.05)$ to the Cox regression model.

\section{RESULTS \\ Differences in baseline characteristics between groups}

Table 1 shows the baseline characteristics of the four study groups. Age was not significantly different between any of the groups ( $\mathrm{p}$ values from 0.16 to 0.72 ). Compared with GP$\mathrm{HF}$, hospital-HF was significantly more associated with male sex, ischaemic heart disease, and atrial fibrillation $(p<0.05)$. Less than $16 \%$ of patients with heart failure received an angiotensin converting enzyme inhibitor.

Cardiac diagnoses in each of the four groups were examined after excluding nursing home residents. The proportional contribution of cardiac diagnoses in the GP-HF group $(n=67)$ was previous myocardial infarction $(10 \%)$, angina pectoris $(6 \%)$, atrial fibrillation $(10 \%)$, valvar heart disease $(4.4 \%)$, pacemaker $(3.0 \%)$, and unknown cause of heart failure $(66 \%)$. The non-HF group $(n=218)$ had slightly more documented ischaemic heart disease: previous myocardial infarction (17\%), angina pectoris (14\%), atrial fibrillation $(7 \%)$, valvar heart disease $(1.4 \%)$, pacemaker $(2.8 \%)$, and unknown heart disease $(58 \%)$ based on an abnormal ECG or chest radiography, or treatment or admission because of possible cardiac symptoms. For the hospital-HF group, the diagnoses were better documented and these were previous myocardial infarction (47\%), angina pectoris $(15 \%)$, atrial fibrillation (15\%), valvar heart disease $(3 \%)$, pacemaker $(3 \%)$, and unknown cause of heart failure $(18 \%)$.

\section{Differences in questionnaire responses between groups}

Table 2 shows that the self reported conditions in the questionnaire also varied between groups. Hospital-HF was associated with the highest frequency of breathlessness, heart disease, and "fluid in the lungs" ( $p<0.05$ ). Although the GP-HF group had a 12 times higher frequency of fluid in the lungs $(p<0.0001)$ than the non-HF patients, the two groups were similar in most other features-for example, breathlessness, heart disease, hypertension, and diabetes. Renal function was not systematically registered.

\section{Long term survival of study groups}

Figure 1 shows the Kaplan-Meier estimated survival curves of the four predefined non-institutionalised study groups. Survival in the GP-HF group was not different from that of the group with non-HF heart disease $(\log$ rank $p=0.173)$, but was significantly better than that of the hospital-HF group ( $\mathrm{p}=0.022$ ). The survival of control participants was significantly better than that of patients with non-HF heart disease ( $p=0.039$ ). The follow up time was from 5.3 to 7.4 years. Five year survival rates were $76 \%$ in control subjects, $71 \%$ in non-HF heart disease, $61 \%$ in GP-HF, and 39\% in hospital-HF. The median survival times were not reached (> 7.4, 7.2, 6.8, and 3.9 years, respectively).

\section{Mortality predictors in the total cohort}

After 7.4 years of follow up 409 deaths were noted in 1979 non-institutionalised patients. Twenty in the control group were lost to follow up (17 emigrated and 3 unknown). A further 109 deaths were registered among 158 nursing home 
Table 1 Clinical features at baseline based on case record reviews, discharge letters, and interviews

\begin{tabular}{|c|c|c|c|c|}
\hline & $\begin{array}{l}\text { Control group } \\
(\%)\end{array}$ & $\begin{array}{l}\text { Non-HF heart } \\
\text { disease }(\%)\end{array}$ & GP-HF (\%) & $\begin{array}{l}\text { Hospital-HF } \\
(\%)\end{array}$ \\
\hline Number & 672 & 242 & 77 & 38 \\
\hline \multicolumn{5}{|l|}{ Demographic } \\
\hline Men & 39 & 35 & 34 & 68 \\
\hline Women & 61 & 65 & 66 & 32 \\
\hline Mean (SD) age (years) & 74.1 (10.9) & 74.4 (10.7) & 75.8 (11.9) & $76.7(10.8)$ \\
\hline \multicolumn{5}{|l|}{ Reaction to questionnaire } \\
\hline Nursing home residency & 15 & 10 & 13 & 11 \\
\hline Non-responder & 15 & 10 & 9 & 11 \\
\hline Responder & 70 & 80 & 78 & 79 \\
\hline \multicolumn{5}{|c|}{ Recorded heart disease in GP case note } \\
\hline Previous MI & 0 & 12 & 6 & 53 \\
\hline Angina pectoris & 0 & 22 & 10 & 45 \\
\hline Atrial fibrillation & 0 & 24 & 27 & 37 \\
\hline Hypertensive heart disease & 0 & 46 & 47 & 32 \\
\hline Diabetic heart disease & 0 & 13 & 12 & 11 \\
\hline \multicolumn{5}{|c|}{ Recorded treatment from GP case notes* } \\
\hline Diuretic agent & 2.2 & 26 & 47 & 55 \\
\hline ASA & 0.3 & 11 & 10 & 37 \\
\hline Digoxin & 0.3 & 24 & 49 & 50 \\
\hline$\beta$ or calcium antagonist & 0.9 & 17 & 9 & 18 \\
\hline ACE inhibitor & 0.3 & 1.7 & 8 & 16 \\
\hline Nitrate agent & 0 & 6.2 & 3 & 21 \\
\hline
\end{tabular}

$A C E$, angiotensin converting enzyme; $A S A$, acetylsalicylic acid; GP, general practice; non-HF, patients with evidence of heart disease but not of heart failure; MI, myocardial infarction.

*Treatment in the control group was not systematically recorded.

residents, which in itself is a surplus mortality (hazard ratio $3.1,95 \%$ confidence interval (CI) 2.5 to 3.8) after adjustment for sex and age. These patients with severe disabilities and comorbidities were excluded from subsequent analyses.

Table 3 shows that mortality was associated with advanced age, male sex, and non-response to the questionnaire. The age and sex adjusted hazard of belonging to a study group was examined in relation to the rest of the healthier patients. The table shows that each study group carried an incremental risk in relation to patients with no or less symptomatic heart disease. As expected the randomly selected control group $(\mathrm{n}=571)$ had a similar age adjusted hazard as the remaining 1088 controls, which indicates that the current difference in patient-control survival would also be seen with an unmatched control sample.

Table 4 shows the value of case note data in predicting mortality in a Cox regression model. Hospital-HF, GP-HF, and non-HF heart disease were all significant predictors after adjustment for sex, age, myocardial infarction, and nonresponse to the questionnaire. Attempts to include angina, atrial fibrillation, diabetic and hypertensive heart disease, and the other variables from table 3 did not add any predictive power.

Table 5 shows the results of a Cox regression model based on patients from the second and third practice who also had responded to the questionnaire. Self reported fluid in the lungs, obstructive airways disease, and breathlessness were the only significant multivariate mortality indicators from the questionnaire apart from sex and age. Curiously enough, none of the variables from table 3 and table 4 added any further predictive power in this selected group of responders.

How to quantify breathlessness is difficult. We explored whether any of the MRC questions were better at predicting death. Each MRC dyspnoea question was included in the model as a replacement for the composite variable of breathlessness based on at least two positive in four dyspnoea questions. The third question, "Do you get breathless when walking on the level in your own pace?", had a slightly higher hazard ratio than the composite variable based on all questions (HR $1.8,95 \%$ CI 1.3 to 2.4 ).

Table 2 Self reported conditions in the questionnaire

\begin{tabular}{|c|c|c|c|c|}
\hline & $\begin{array}{l}\text { Control group } \\
(\%)\end{array}$ & $\begin{array}{l}\text { Non-HF heart } \\
\text { disease (\%) }\end{array}$ & $\begin{array}{l}\text { GP-HF } \\
(\%)\end{array}$ & $\begin{array}{l}\text { Hospital-HF } \\
(\%)\end{array}$ \\
\hline Questions asked in three general practices & $(n=469)$ & $(n=193)$ & $(n=60)$ & $(n=30)$ \\
\hline Breathless by 2 in 4 questions & 19 & 49 & 48 & 87 \\
\hline Breathless walking at own pace & 9.0 & 25 & 27 & 43 \\
\hline Any heart disease & 2.1 & 51 & 47 & 80 \\
\hline Questions asked in two general practices & $(n=347)$ & $(n=156)$ & $(n=37)$ & $(n=19)$ \\
\hline $\mathrm{MI}$ & 0.3 & 12 & 14 & 47 \\
\hline Fluid in the lungs & 0.3 & 2.6 & 38 & 84 \\
\hline Peripheral fluid retention & 13 & 18 & 46 & 63 \\
\hline Hypertension & 18 & 50 & 54 & 21 \\
\hline Diabetes & 2.9 & 13 & 8 & 16 \\
\hline Asthma or bronchitis & 14 & 18 & 32 & 32 \\
\hline
\end{tabular}




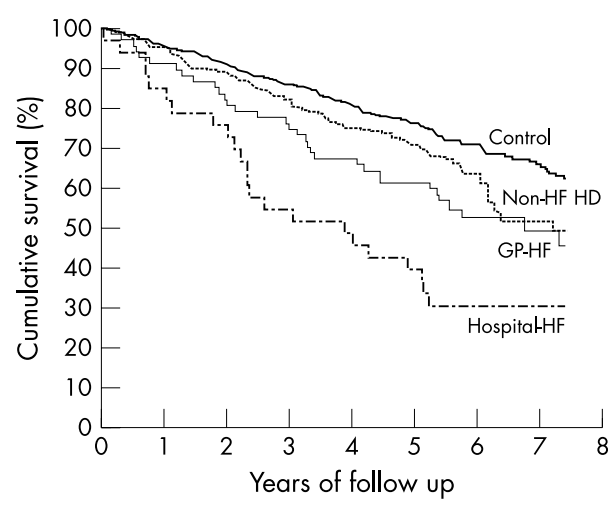

Figure 1 Kaplan-Meier estimated survival curves after exclusion of nursing home residents. The number of deaths were 175 in 571 control participants (Control), 82 in 218 patients with non-heart failure heart disease (Non-HF HD), 33 in 67 patients thought to have heart failure in general practice (GP-HF), and 24 in 34 patients with heart failure managed in hospital (Hospital-HF).

\section{DISCUSSION}

\section{Main results}

An important novel finding is that patients in the GP-HF group had a worse survival than controls, a better prognosis than those with hospital-HF, and a similar survival to nonHF patients. The poorer survival in hospitalised patients is most likely to be caused by them having more definite and severe heart failure. Other significant mortality indicators were advanced age, male sex, suspected heart failure and heart disease in both general practice and hospitals, a history of myocardial infarction, obstructive airways disease, and breathlessness when patients walk at their own pace.
Table 4 Long term multivariate risk factors of mortality from general practice case notes

\begin{tabular}{llll}
\hline & HR & 95\% Cl & p Value \\
\hline Sex (0-woman. 1-man) & 2.1 & 1.7 to 2.6 & $<0.0001$ \\
Age (per 10 years) & 2.4 & 2.2 to 2.6 & $<0.0001$ \\
Non-responder status & 2.0 & 1.5 to 2.5 & $<0.0001$ \\
Hospital-HF & 2.1 & 1.3 to 3.5 & 0.0021 \\
GP-HF & 1.7 & 1.2 to 2.5 & 0.0035 \\
Non-HF heart disease & 1.4 & 1.0 to 1.8 & 0.0270 \\
Previous MI & 1.6 & 1.0 to 2.5 & 0.0355 \\
\hline
\end{tabular}

Data based on multivariate Cox modelling in 1979 subjects residing outside nursing homes. The HR is associated with recordings in the general practice's case notes and whether the patient had responded to the questionnaire. Other evaluated variables in that model were not significant-for example, angina, ischaemic heart disease (angina or MI), atrial fibrillation, hypertension, or diabetes.

\section{Other studies}

Any discussion of the possible reasons for differences in survival data between studies are hampered by the large heterogeneities between study designs. The five year survival rate of $61 \%$ in the present group of patients with heart failure diagnosed by general practitioners is completely in line with $59 \%$ in the Rotterdam population study. ${ }^{1}$ That survival rate was, however, worse than in the Gothenburg and NHANES study possibly because the patients were older and more rigorously defined.

The $39 \%$ five year survival in the present patients with heart failure diagnosed in hospital is slightly better than the $35 \%$ observed in Olmsted county, ${ }^{6}$ and $25 \%$ for men and $38 \%$ for women in the Framingham study, perhaps because the present study included mainly patients with prevalent disease rather than patients with a new onset of heart failure. This explanation is supported by several other

Table 3 Long term mortality risk factors adjusted for sex and age

\begin{tabular}{|c|c|c|c|c|}
\hline & $\mathbf{N}$ & HR & $95 \% \mathrm{Cl}$ & p Value \\
\hline \multicolumn{5}{|l|}{ Demographic data } \\
\hline Sex (0-woman, 1-man) & 1979 & 2.11 & 1.73 to $2.58^{*}$ & $<0.0001$ \\
\hline Age (per 10 years) & 1979 & 2.56 & 2.35 to $2.80^{*}$ & $<0.0001$ \\
\hline No response to questionnaire & 1979 & 1.77 & 1.40 to $2.24^{*}$ & $<0.0001$ \\
\hline \multicolumn{5}{|c|}{ Study groups (group $v$ rest of the participants) } \\
\hline Hospital-HF (34 v 1945) & 1979 & 2.02 & 1.31 to $3.10^{*}$ & 0.0014 \\
\hline GP-HF (67 v 1878) & 1945 & 1.60 & 1.11 to $2.30^{*}$ & 0.0108 \\
\hline Non-HF $(218$ v 1660) & 1878 & 1.31 & 1.01 to $1.69^{*}$ & 0.0381 \\
\hline Control group (571 v 1089) & 1660 & 1.05 & 0.78 to 1.41 & 0.7657 \\
\hline \multicolumn{5}{|c|}{ Recorded heart disease in general practice case notes } \\
\hline Previous Ml & 1979 & 2.13 & 1.43 to $3.16^{*}$ & 0.0002 \\
\hline Angina pectoris & 1979 & 1.64 & 1.17 to $2.31^{*}$ & 0.0043 \\
\hline Atrial fibrillation & 1979 & 1.36 & 0.97 to 1.90 & 0.0710 \\
\hline Hypertensive heart disease & 1979 & 1.06 & 0.80 to 1.43 & 0.6732 \\
\hline Diabetic heart disease & 1979 & 1.27 & 0.77 to 2.09 & 0.3548 \\
\hline \multicolumn{5}{|c|}{ Questions asked in three general practices } \\
\hline Breathless by 2 in 4 questions & 1700 & 2.12 & 1.68 to $2.66^{*}$ & $<0.0001$ \\
\hline Breathless walking at own pace & 1700 & 2.58 & 1.99 to $3.35^{*}$ & $<0.0001$ \\
\hline Any heart disease & 1700 & 1.39 & 1.05 to $1.83^{*}$ & 0.0196 \\
\hline \multicolumn{5}{|c|}{ Questions asked in two general practices } \\
\hline $\mathrm{Ml}$ & 901 & 2.1 & 1.3 to $3.5^{*}$ & 0.0047 \\
\hline Fluid in the lungs & 901 & 2.5 & 1.6 to $4.0^{*}$ & $<0.0001$ \\
\hline Peripheral fluid retention & 901 & 1.8 & 1.3 to $2.5^{*}$ & 0.0007 \\
\hline Hypertension & 901 & 1.2 & 0.9 to 1.7 & 0.2507 \\
\hline Diabetes & 901 & 1.6 & 0.9 to 2.6 & 0.0804 \\
\hline Asthma or bronchitis & 901 & 2.0 & 1.4 to $2.8^{*}$ & 0.0002 \\
\hline
\end{tabular}

The sample size (N) was reduced from 2157 to 1979 after excluding 158 nursing home residents and 20 controls with missing end point values. When examining the effect of a study group, $\mathrm{N}$ was reduced by subsequent exclusion of 34 from the hospital-HF group, 67 from the GP-HF group, and 218 from the non-HF group. The questionnaire variables were analysed only for responders $(n=1700)$ and some questions only applied to 901 participants.

${ }^{*} \mathrm{p}<0.05$.

$\mathrm{Cl}$, confidence interval; $\mathrm{HR}$, hazard ratios for predictors of mortality in the Cox model. 
Table 5 Long term multivariate risk factors of mortality from the questionnaire

\begin{tabular}{lllr}
\hline & HR & 95\% Cl & p Value \\
\hline Sex (0-woman. 1-man) & 2.2 & 1.6 to 3.0 & $<0.0001$ \\
Age (per 10 years) & 2.5 & 2.1 to 2.9 & $<0.0001$ \\
Fluid in the lungs & 1.7 & 1.0 to 2.7 & 0.0404 \\
Breathless walking at own pace & 2.2 & 1.6 to 3.1 & $<0.0001$ \\
Asthma or bronchitis & 1.5 & 1.0 to 2.2 & 0.0296
\end{tabular}

Multivariate Cox model in 901 subjects who had responded to the questionnaire and were residing outside nursing homes. The HR is associated with self reported conditions in the questionnaire. Other questionnaire variables in that model were not significant-for example, breathless in two of four questions, peripheral fluid retention, hypertension, diabetes, any heart disease, or MI. The model was not improved by inclusion of general practice case note variables such as hospital-HF, GP-HF, non-HF heart disease, or a previous MI.

studies: the five year survival rate was only $25 \%$ in patients with a first hospitalisation for heart failure in Scotland ${ }^{14}$; new onset of heart failure, when validated by cardiologists, has a $25 \%$ mortality within the first three months; $s^{5}$ and patients who survived the first 90 days in the Framingham heart study had a median survival time of 3.2 years in men and 5.4 years in women. ${ }^{15}$

\section{Strengths and limitations}

The present study design provided unique information on unselected patients with heart failure in the community. Such patients are not included in clinical trials and population surveys. The Boston scoring system used here applies to patients with a definite as well as a possible diagnosis of heart failure. ${ }^{9}$ It was based on clinicoradiographic criteria, rather than echocardiographic evidence. Using this will obviously lead to the inclusion of some patients with a less severe and less definite diagnosis of heart failure. We have in an earlier publication reported that GPHF patients had a $20 \%$ frequency of left ventricular systolic dysfunction (ejection fraction $<0.45$ ), 30\% had other cardiac abnormalities, and half had no major cardiac abnormality. ${ }^{9}$ In contrast half the patients with hospital-HF had left ventricular systolic dysfunction and the remainder had some other cardiac abnormality.

Nursing home residents in Denmark are characterised by multiple mental and physical disabilities. The survival data of the present study were not biased by the surplus mortality that is seen in such very old patients. Furthermore, the documentary level for nursing home patients was generally poorer than for other patients with regard to both case note and questionnaire data. The purpose of a matched control group was to illustrate the effect of suspected heart failure independent of age, sex, and general practice connection. The cardiovascular risk factors would perhaps be more powerful if we had used cardiovascular deaths instead of all cause mortality, but that would have led to fewer and more uncertain end points.

End points were missing for 20 control participants. Seventeen patients had migrated and three had disappeared, and the median age of migrating patients was 46 years. The fate of these low risk patients is unlikely to disturb the overall conclusion of this study.

\section{Perspective}

Clinical features, especially sex, and survival of GP-HF patients are clearly different from those in hospital and clinical trials. Even though the suspicion of heart failure, diagnosed from clinical observations alone, may be doubtful in about half the cases ${ }^{9}$ it identifies a patient group with a significant risk per se. In this paper we point out some clues that may help general practitioners identify patients with the poorest survival. It would be logical to use diagnostic cardiopulmonary tests in these patients to see whether treatable conditions exist, although cost effectiveness would have to be examined in a prospective controlled trial. Once patients at risk are singled out, further diagnostic and prognostic stratification is warranted by plasma concentrations of natriuretic peptides, echocardiography, and the ECG. ${ }^{16-18}$

\section{ACKNOWLEDGEMENTS}

This study was supported by the Danish Heart Foundation. We thank the Cardiology Department, Hvidovre Copenhagen University Hospital for housing the base line examination of the cohort. We thank Professor Christian Torp-Petersen for retrieving the survival data.

\section{Authors' affiliations}

O Wendelboe Nielsen, J Fischer Hansen, Cardiology Department,

Bispebjerg Hospital, University of Copenhagen, Copenhagen, Denmark J Hilden, Department of Biostatistics, University of Copenhagen

T McDonagh, Cardiology Department, The Western Infirmary, Glasgow and MRC Clinical Research Initiative in Heart Failure, Glasgow University, Glasgow, UK

\section{REFERENCES}

1 Mosterd A, Cost B, Hoes AW, et al. The prognosis of heart failure in the general population. The Rotterdam study. Eur Heart J 2001;22:1318-27.

2 Wilhelmsen L, Rosengren A, Eriksson $\mathrm{H}$, et al. Heart failure in the general population of men: morbidity, risk factors and prognosis. J Intern Med 2001;249:253-61.

3 Schocken DD, Arrieta MI, Leaverton PE, et al. Prevalence and mortality rate of congestive heart failure in the United States. J Am Coll Cardiol 1992;20:301-6.

4 Kannel WB. Epidemiology and prevention of cardiac failure: Framingham study insights. Eur Heart J 1987;8(suppl F):23-6.

5 Cowie MR, Wood DA, Coats AJ, et al. Survival of patients with a new diagnosis of heart failure: a population based study. Heart 2000;83:505-10.

6 Senni M, Tribouilloy CM, Rodeheffer RJ, et al. Congestive heart failure in the community: a study of all incident cases in Olmsted County, Minnesota, in 1991. Circulation 1998:98:2282-9.

7 Remes J, Miettinen H, Reunanen A, et al. Validity of clinical diagnosis of heart failure in primary health care. Eur Heart J 1991;12:315-21.

8 Cowie MR, Wood DA, Coats AJ, et al. Incidence and aetiology of heart failure; a population-based study. Eur Heart J 1999;20:421-8.

9 Nielsen OW, Hilden J, Larsen CT, et al. Cross sectional study estimating prevalence of heart failure and left ventricular systolic dysfunction in community patients at risk. Heart 2001;86:172-8.

10 Davies M, Hobbs F, Davis R, et al. Prevalence of left-ventricular systolic dysfunction and heart failure in the echocardiographic heart of England screening study: a population based study. Lancet 2001;358:439-44.

11 Nielsen OW, Hansen JF, Hilden J, et al. Risk assessment of left ventricular systolic dysfunction in primary care: cross sectional study evaluating a range of diagnostic tests. BMJ 2000;320:220-4.

12 Rose GA, Blackburn H. Cardiovascular survey methods. Monogr Ser World Health Organ 1968:56:1-188.

13 Carlson KJ, Lee DC, Goroll AH, et al. An analysis of physicians' reasons for prescribing long-term digitalis therapy in outpatients. J Chronic Dis 1985;38:733-9.

14 Stewart S, Maclntyre K, Hole DJ, et al. More 'malignant' than cancer? Fiveyear survival following a first admission for heart failure. Eur J Heart Fail $2001 ; 3: 315-22$.

15 Kannel WB, Ho K, Thom T. Changing epidemiological features of cardiac failure. Br Heart J 1994;72(2 suppl):S3-9.

16 Nielsen OW, Hilden J, Hansen JF. Strong prognostic value of combining Nterminal atrial natriuretic peptide and ECG to predict death in heart patients from general practice. Heart 2001;86:218-9.

17 McDonagh TA, Cunningham AD, Morrison CE, et al. Left ventricular dysfunction, natriuretic peptides, and mortality in an urban population. Heart 2001;86:21-6.

18 Bettencourt $P$, Ferreira A, Dias $P$, et al. Predictors of prognosis in patients with stable mild to moderate heart failure. J Card Fail 2000;6:306-13. 